DOI 10.15593/2224-9354/2018.2.23

УДК 338.583

\author{
Е.В. Антоненко, М.А. Полякова, С.А. Федосеев
}

ОЦЕНКА СТЕПЕНИ СОГЛАСОВАННОСТИ
ТРЕБОВАНИЙ ПОТРЕБИТЕЛЯ И ИЗГОТОВИТЕЛЯ КАК МЕТОД СОКРАЩЕНИЯ ТРАНСАКЦИОННЫХ ИЗДЕРЖЕК

\begin{abstract}
Предложено определение трансакционных издержек, возникающих в результате рыночного взаимодействия предприятий и включающих реальные и альтернативные потери, понесенные предприятиями в результате такого взаимодействия. Рассмотрены трансакционные издержки, связанные с ведением переговоров между потребителем и изготовителем металлопродукции. Отмечено, что на практике время, затрачиваемое потребителем и изготовителем металлопродукции на переговоры по согласованию требований, является весьма значительным. Поэтому актуальной является задача поиска подходов для сокращения продолжительности подобных переговоров и экономии связанных с этими переговорами трансакционных издержек. Рассмотрен один из таких подходов, базирующийся на применении количественного критерия, оценивающего степень согласованности требований, обсуждаемых потребителем и изготовителем. В качестве одного из методов определения такого критерия предложено использование математических моделей, основанных на применении S-образных кривых и аппарата квалиметрии. Показано, что для разработки математических моделей, позволяющих количественно оценить степень согласованности требований потребителя и изготовителя продукции в ходе ведения переговоров, необходимо учитывать особенности нормирования показателей качества продукции в нормативной и технической документации. Сформулированы принципы количественной оценки степени достижения соглашения между потребителем и изготовителем, а именно: оценка должна рассчитываться как функция от некоторого показателя качества продукции; оценка должна непрерывно зависеть от значения показателя качества продукции; оценка может иметь характерные участки интенсивного и замедленного роста. Приведен пример того, как предложенные методы количественной оценки степени согласованности требований позволяют существенно сократить время переговоров между потребителем и изготовителем металлопродукции и, как следствие, уменьшить потери потребителя, связанные с повышением цен изготовителем.

Ключевые слова: трансакционные издержки, сокращение потерь, математические модели, качество продукции, степень согласованности требований.
\end{abstract}

Tрансакционные издержки. Трансакционные издержки пронизывают все уровни отношений: между людьми, предприятиями, корпорациями. Производя поиск будущих партнеров, подписывая контракты и исполняя заключенные соглашения, предприятия сталкиваются с сопутствующими затрата-

(C) Антоненко Е.В., Полякова М.А., Федосеев С.А., 2018

Антоненко Елизавета Викторовна - преподаватель кафедры «Таможенное дело» ФГАОУ ВО «Южно-Уральский государственный университет (национальный исследовательский университет)», e-mail: Elizaveta.antonenko@susu.ru.

Полякова Марина Андреевна - канд. техн. наук, доцент кафедры «Технологии обработки материалов» ФГБОУ ВО «Магнитогорский государственный технический университет им. Г.И. Носова», e-mail: m.polyakova-64@mail.ru.

Федосеев Сергей Анатольевич - д-р техн. наук, профессор кафедры «Вычислительная математика и механика» ФГБОУ ВО «Пермский национальный исследовательский политехнический университет», e-mail: fsa@gelicon.biz. 
ми. Они могут быть весьма значительны по величине, что снижает эффективность управления предприятиями.

Единого общепризнанного определения понятия «трансакционные издержки» на сегодняшний день не существует [1-4]. Понимание данного термина зависит от общей позиции исследователя, от цели его работы, области исследования, принадлежности к той или иной экономической школе. В рамках настоящей статьи будем использовать определение, предложенное в работе [5]: «Трансакционные издержки - это издержки, возникающие в результате рыночного взаимодействия предприятия с внешней средой при решении проблем, вызванных асимметричностью информации, административным взаимодействием, а также реальные и альтернативные потери, понесенные предприятием в результате данного взаимодействия».

По аналогии с определением, на сегодняшний день не существует и общепринятой классификации трансакционных издержек. Наиболее распространенной и относительно полной можно считать классификацию НортаЭггертссона (первым ее предложил Норт, более полно сформулировал Эггертссон) [6]. Данная классификация представлена на рис. 1.

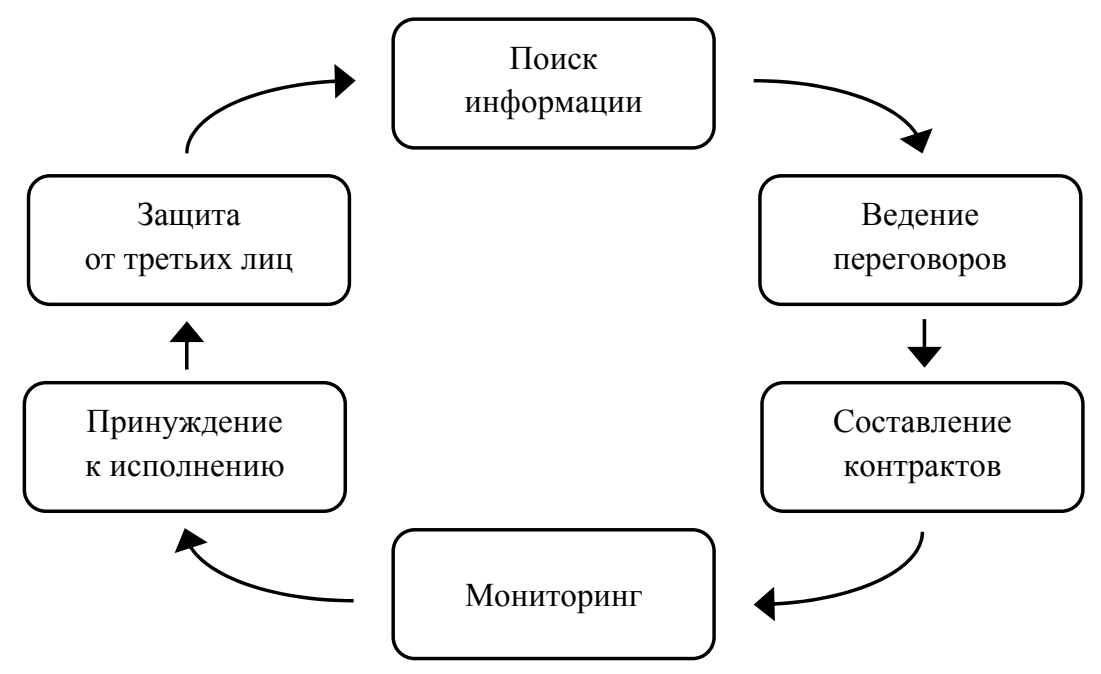

Рис. 1. Классификация трансакционных издержек Норта-Эггертссона

Исследования даже простейших переговорных задач показывают, что они не всегда приводят к оптимальным решениям. В то время как стороны переговоров считают, что они успешно провели переговоры, последующая оценка их результата показывает, что они были не оптимальны. Движение к оптимальному решению состоит в получении дополнительных выгод обеими сторонами, поэтому переговоры можно рассматривать с позиции решения задач поиска консенсуса [7]. 
Исследования по многоаспектным переговорам позволили выделить три основных способа достижения консенсуса [8-10]: package deal (все аспекты рассматриваются единым целым), параллельная процедура (все аспекты обсуждаются одновременно, но независимо друг от друга), последовательная процедура (все аспекты обсуждаются последовательно). Среди указанных именно процедура package deal хорошо работает в ситуациях, когда переговорщики должны достигнуть соглашения быстро [11].

В рамках настоящей статьи будут рассмотрены трансакционные издержки, связанные с ведением переговоров между потребителем и изготовителем металлопродукции. В ходе ведения переговоров каждая сторона отстаивает собственную точку зрения и стремится к удовлетворению своих интересов по регламентации в нормативной и технической документации необходимых значений показателей качества металлоизделия, что в значительной мере усложняет процесс достижения соглашения. На практике время, затрачиваемое потребителем и изготовителем металлопродукции на переговоры по согласованию требований, является весьма значительным и может составлять от 6 до 12 месяцев. Поэтому актуальной является задача поиска подходов для сокращения продолжительности подобных переговоров и, как следствие, значительной экономии связанных с этими переговорами трансакционных издержек. Один из таких подходов базируется на применении количественного критерия, который бы позволил сторонам быстрее и точнее оценить степень согласованности обсуждаемых требований.

Математические модели для измерения степени согласованности требований потребителя и изготовителя. Для разработки математических моделей, позволяющих количественно оценить степень согласованности требований потребителя и изготовителя продукции в ходе ведения переговоров, необходимо разработать систему критериев с учетом особенностей нормирования показателей качества продукции в нормативной и технической документации: номинальным или интервальным значением.

Основной принцип оценивания степени согласованности требований потребителя и изготовителя заключается в приоритете требований потребителя. В дальнейшем оценку степени близости требований потребителя и изготовителя будем обозначать буквой $M$, которая может принимать значения в интервале $[0 ; 1]$. Такая шкала традиционно используется в квалиметрии при оценке показателей качества продукции [12] и характеризуется высокой аналитической надежностью.

Пусть состоянию «полная неудовлетворенность» соответствует значение 0 оценки $M$, а состоянию «полная удовлетворенность»- значение 1 оценки $M$. Если $F$ - множество значений показателя качества продукции, определенное изготовителем, а $U$ - множество значений показателя качества продукции, определенное потребителем, тогда предложенные соответствия математически можно представить следующим образом: 
1. Если $F \subset U$, то $M=1$, т.е. если требования потребителя перекрываются требованиями изготовителя, то значение оценки равно 1.

2. Если $F \cap U=\varnothing$, то $M=0$, т.е. при полном несовпадении требований потребителя и изготовителя значение оценки равно 0.

3. $F \cap U \neq \varnothing$ и $F \notin U$, то $M=\frac{V(F \cap U)}{V(F)}, 0<M<1$,

где $V(X)$ - некоторая количественная мера для множества значений $X$ показателя качества продукции.

С учетом особенностей процесса ведения переговоров между потребителем и изготовителем, в ходе которого происходит согласование значений показателей качества продукции, могут быть сформулированы принципы количественной оценки достижения соглашения [13]:

1. Оценка степени согласованности требований потребителя и изготовителя рассчитывается как функция от некоторой величины в зависимости от вида нормирования показателя качества продукции. Если показатель качества нормируется номинальным значением, то оценка степени согласованности требований сторон рассчитывается в зависимости от величины расхождения между значениями данного показателя, задаваемыми потребителем и изготовителем. Если показатель качества нормируется в виде интервала значений, то оценка степени согласованности требований сторон рассчитывается в зависимости от величины интервала пересечения требований потребителя и изготовителя по значениям показателя.

2. Непрерывность изменения оценки степени согласованности требований потребителя и изготовителя от изменения значения показателя качества продукции. Небольшое изменение показателя качества изделия, которое происходит в ходе ведения переговоров между потребителем и изготовителем при согласовании его значения, не вызывает скачкообразного изменения величины оценки.

3. Изменение оценки степени согласованности требований потребителя и изготовителя имеет два характерных этапа: на первом этапе изменение оценки увеличивается более интенсивно до некоторой определенной величины, после которой начинается этап замедленного увеличения оценки до максимального значения, означающего достижение соглашения между потребителем и изготовителем.

Тогда для моделирования оценки степени согласованности требований потребителя и изготовителя (степени достижения консенсуса) могут быть применены возрастающая и убывающая S-образные кривые (рис. 2), математическое описание которых приведено в работах [14-16]. При этом вид

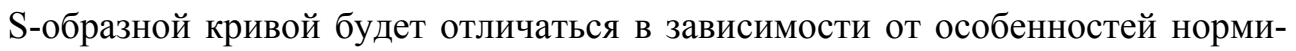
рования единичного показателя качества, по численному значению которого идет процесс переговоров между потребителем и изготовителем. 

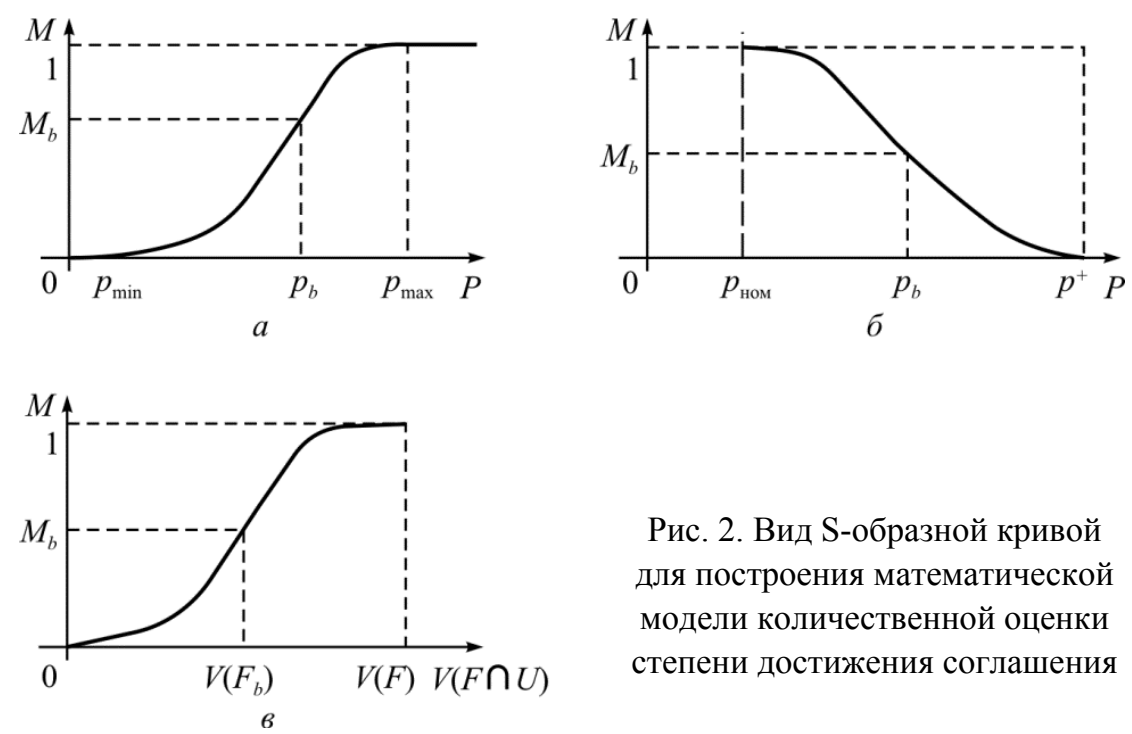

\begin{abstract}
Рис. 2. Вид S-образной кривой для построения математической модели количественной оценки степени достижения соглашения
\end{abstract}

На рис. 2, а представлена возрастающая S-образная кривая, описывающая оценку степени близости требований потребителя и изготовителя при задании единичного показателя номинальным значением с минусовым допуском. На рис. 2, б представлена убывающая S-образная кривая, описывающая оценку степени близости требований потребителя и изготовителя при задании единичного показателя номинальным значением с плюсовым допуском. На рис. 2, в представлена возрастающая S-образная кривая, описывающая оценку степени близости требований потребителя и изготовителя при задании единичного показателя интервальным значением.

На рис. 2 приняты следующие обозначения: $M$ - значение оценки степени согласованности требований потребителя и изготовителя; $P$ - оцениваемое значение единичного показателя; $p_{\min }$ и $p_{\max }-$ наименьшее и наибольшее значение единичного показателя соответственно; $p_{b}$ - точка, делящая кривую на участки увеличения и уменьшения скорости изменения значения оценки; $M_{b}$ - значение оценки в точке $p_{b} ; p_{\text {ном }}-$ номинальное значение единичного показателя; $p^{+}-$значение плюсового допуска; $V(F)$ - некоторая количественная мера интервала изготовителя; $V\left(F_{b}\right)$ - значение меры в точке, делящей кривую на участки увеличения и уменьшения скорости изменения значения оценки; $V(F \cap U)$ - мера пересечения интервалов значений потребителя и изготовителя; $M_{V\left(F_{b}\right)}$ - значение оценки в точке $V\left(F_{b}\right)$.

Поскольку показатели качества, по численным значениям которых идет процесс согласования, могут быть доминирующими или компенсируемыми, то для вычисления полной оценки можно воспользоваться формулой расчета комплексной оценки, используемой в квалиметрии $[14,17]$ : 


$$
C=\frac{\prod_{i=1}^{m} d_{i}^{1 / m}\left(d_{i}+1\right)^{\alpha_{i}} \prod_{j=1}^{n}\left(k_{j}+1\right)^{\beta_{j}}}{4}
$$

где $d_{i}$ - доминирующий показатель; $k_{j}$ - компенсируемый показатель; $m$ и $n$ - число соответственно доминирующих и компенсируемых показателей; $\alpha_{i}$ и $\beta_{j}-$ весомости соответственно доминирующих и компенсируемых показателей, причем

$$
\sum_{i=1}^{m} \alpha_{i}=\sum_{j=1}^{n} \beta_{j}=1
$$

Пример оценок степени согласованности требований потребителя и изготовителя представлен в табл. 1 [13].

Таблица 1

Оценка степени согласованности требований потребителя и изготовителя для случая, когда диапазон требований потребителя лежит внутри диапазона требований изготовителя

\begin{tabular}{|l|c|c|c|c|}
\hline $\begin{array}{c}\text { Выражение единичного } \\
\text { показателя }\end{array}$ & $\begin{array}{c}\text { Требование } \\
\text { потребителя }\end{array}$ & $\begin{array}{c}\text { Требование } \\
\text { изготовителя }\end{array}$ & Оценка & $\begin{array}{c}\text { Полная } \\
\text { оценка }\end{array}$ \\
\cline { 1 - 4 } Номинальное значение & 190 & 200 & Локальная 0,92 & \multirow{2}{*}{0,78} \\
\cline { 1 - 4 } Интервальное значение & $100-200$ & $20-250$ & Интервальная 0,76 & \\
\hline
\end{tabular}

Пример сокращцения трансакционных издержек ведения переговоров. Определим возможный экономический эффект от применения математических моделей количественной оценки степени согласованности требований потребителя и изготовителя на примере разработки нормативного документа на болт машиностроительный [18]. Если данный вид крепежного изделия будет иметь более высокий уровень механических свойств, необходимых потенциальному потребителю, по сравнению со значениями свойств по ГОСТ 7798-70 «Болты с шестигранной головкой класса точности В. Конструкция и размеры», то потребуется проведение переговоров между потребителем и изготовителем для согласования численных значений соответствующих единичных показателей изделия. Продолжительность данных переговоров может быть сокращена за счет быстрого и достаточно точного измерения степени согласованности требований сторон на основе описанных выше математических моделей.

Для оценки экономического эффекта используем данные годового отчета ОАО «ММК-МЕТИЗ» за 2014 год, утвержденного годовым общим собранием акционеров 5 июня 2015 года, и цены на производимую продукцию по данным ООО «Торговый дом «Метиз» по состоянию на 01.12.2015 [19, 20]. 
Для проведения расчетов используем следующие исходные данные:

1) объем производства крепежа общего назначения составляет 26400 т в год;

2) доля нового крепежа общего назначения в структуре всей производимой металлопродукции составляет $5 \%$;

3) медианная цена на болт машиностроительный (класс прочности 8.8, диаметр 16 мм, длина 45 мм) составляет 62600 руб/т.;

4) с учетом данных независимой аналитической компании Alto Consulting Group [21] о динамике цен на металлопродукцию в период 2012-2015 годов, средние цены производителей на крепежные изделия (болты, гайки, винты) повышаются на 9,3 \% в год.

Результаты расчета экономического эффекта от сокращения продолжительности переговоров при согласовании требований потребителя и изготовителя приведены в табл. 2.

Таблица 2

Оценка экономической эффективности от ускорения поставки продукции

\begin{tabular}{|l|c|l|}
\hline \multicolumn{1}{|c|}{ Наименование } & $\begin{array}{l}\text { Численное } \\
\text { 3начение }\end{array}$ & \multicolumn{1}{|c|}{ Пояснение } \\
\hline $\begin{array}{l}\text { Объем производства нового кре- } \\
\text { пежа, т в год }\end{array}$ & 1320 & $\begin{array}{l}\text { Значение, полученное умножением } \\
\text { объема производства на долю нового } \\
\text { крепежа }(26 \text { 400×0,05) }\end{array}$ \\
\hline $\begin{array}{l}\text { Прибавка к стоимости при подо- } \\
\text { рожании нового крепежа на 9,3\%, } \\
\text { тыс. руб. за 1 тонну }\end{array}$ & 5,8 & $\begin{array}{l}\text { Значение, полученное умножением ме- } \\
\text { дианной цены на подорожание стоимо- } \\
\text { сти нового крепежа (62,6×0,093) }\end{array}$ \\
\hline $\begin{array}{l}\text { Выручка от выпуска нового кре- } \\
\text { пежа, тыс. руб. в год }\end{array}$ & 7656 & $\begin{array}{l}\text { Значение, полученное умножением } \\
\text { объема производства нового крепежа } \\
\text { на прибавку в стоимости }(1320 \times 5,8)\end{array}$ \\
\hline $\begin{array}{l}\text { Экономия потребителя, получен- } \\
\text { ная от ускоренного выпуска ново- } \\
\text { го крепежа, тыс. руб. в месяц }\end{array}$ & 638 & $\begin{array}{l}\text { Значение, полученное делением вы- } \\
\text { ручки от выпуска нового крепежа на } \\
12 \text { месяцев }(7656 / 12)\end{array}$ \\
\hline
\end{tabular}

На основании данных, приведенных в табл. 2, можно сделать следующий вывод. Если благодаря предложенной математической модели ускорить процесс согласования требований потребителя и изготовителя, то потребитель сможет сократить потери на 638 тыс. руб. в месяц за счет получения продукции до увеличения цены со стороны изготовителя. Данное обстоятельство, несомненно, положительным образом отразится на удовлетворенности потребителя и повысит его лояльность к изготовителю.

Заключение. Трансакционные издержки ведения переговоров между потребителями и изготовителями металлопродукции на данный момент весьма значительны. Один из подходов, направленных на сокращение подобных издержек, базируется на использовании математических моделей, основанных на применении S-образных кривых и аппарата квалиметрии. Данный подход позволяет количественно оценить степень согласованности требований потребителя и изготовителя 
металлопродукции и, как следствие, существенно сократить время согласования требований потребителя и изготовителя. В результате потребитель может заметно сократить потери, связанные с повышением цен изготовителем.

\section{Список литературы}

1. Wallis J.J. Measuring the Transaction Sector in the American Economy. 1870-1970. // Long-Term Factors in American Economic Growth. - Chicago, 1986. - P. 95-162.

2. Cheung S.N.S. Transaction Costs, Risk Aversion, and the Choice of Contractual Arrangements // Journal of Law \& Economics. - 1969. - Vol. 12, no. 1. - P. 23.

3. Williamson O.E. Transaction-cost economics: The governance of contractual relations // Journal of Law and Economics. - 1979. - Vol. 22, no. 2. P. 233-261.

4. Jensen M.C., Meckling W.H. Theory of the firm: Managerial behavior, agency costs and ownership structure // Journal of Financial Economics. - 1976. Vol. 3, no. 4. - P. 305-360.

5. Антоненко Е.В. К раскрытию сущности и классификации трансакционных издержек // Вестник ЮУрГУ. Экономика и менеджмент. - 2015. - Т. 9, № $1 .-$ C. $74-80$.

6. Eggertsson T. The role of transaction costs and property rights in economic analysis // European Economic Review. - 1990. - Vol. 34, no. 2/3. - P. 450-457.

7. Prietula M.J., Weingart L.R. Negotiation Offers and the Search for Agreement // Negotiation and Conflict Management Research. - 2011. - Vol. 4, № 2. - P. 77-109.

8. Robu V., Somefun D.J.A., Poutre J.L. Modeling complex multi-issue negotiations using utility graphs // Proc. of the Forth International Joint Conference on Autonomous Agents and Multi-Agent Systems. - Utrecht, 2005. - P. 280-287.

9. Fujita K., Ito T., Klein M. An approach to scalable multi-issue negotiation: Decomposing the contract space // Computation intelligence. - 2014. - Vol. 30. P. 30-47.

10. Abedin F., Chao K.-M., Godwin N. An agenda based multi issue negotiation approach // Journal of Ambient Intelligence and Humanized Computing. - 2014. - Vol. 5, no. 4. - P.455-473.

11. Fatima S.S., Wooldridge M., Jennings N.R. Multi-issue negotiation with deadlines // Journal of Artificial Intelligence Research. - 2006. - Vol. 27. P. 381-417.

12. Азгальдов Г.Г., Костин А.В., Садовов В.В. Квалиметрия для всех. М.: ИнформЗнание, 2012. - 165 с.

13. Полякова М.А. Развитие теории оценки согласованности технических требований на металлопродукцию при разработке нормативной и технической документации: дис. ... д-ра техн. наук: 05.02.23 / Магнит. гос. техн. ун-т им. Г.И. Носова. - Магнитогорск, 2017. - 235 с. 
14. Полякова М.А. Особенности применения математических моделей для оценки степени достижения консенсуса при разработке требований стандартов // Международный научно-исследовательский журнал. - 2016. № 11 (53), ч. 4. - С. 104-109.

15. Рубин Г.Ш., Касаткина Е.Г. S-образная математическая модель единичной оценки качества // Качество в обработке материалов. - 2014. - № 2. C. $74-81$.

16. Рубин Г. Ш., Полякова М.А., Гун Г.С. Моделирование технологического трансформирования на основе S-образных кривых развития // Вестник Магнитогорского государственного технического университета им. Г.И. Носова. - 2015. - № 1. - С. 70-75.

17. Азгальдов Г.Г. Теория и практика оценки качества товаров. Основы квалиметрии. - М.: Экономика, 1982. - 256 с.

18. Данилова Ю.В. Совершенствование процедуры разработки стандартов на металлоизделия формализацией согласования позиций заинтересованных сторон: дис. ... канд. техн. наук: 05.02.23 / МГТУ им. Г.И. Носова. Магнитогорск, 2016. - 155 с.

19. ММК-МЕТИЗ [Электронный ресурс]. - URL: http://mmk-metiz.ru (дата обращения: 02.12.2014).

20. ММК-МЕТИЗ. Акционерам и инвесторам [Электронный ресурс]. URL: http://www.mmk-metiz.ru/investor/reports/ (дата обращения: 7.12.2015).

21. Alto Consulting Group [Электронный pecypc]. - URL: http:// alto-group.ru/otchot/marketing/363-rynok-metizov-krepezhnyx-izdelij-tekushhayasituaciya-i-prognoz-2014-2018-gg.html (дата обращения: 5.12.2015).

\section{References}

1. Wallis J.J., North D.C. Measuring the transaction sector in the American economy, 1870-1970. Long-Term Factors in American Economic Growth, 1986, pp. 95-162.

2. Cheung S.N.S. Transaction costs, risk aversion, and the choice of contractual arrangements. Journal of Law \& Economics, 1969, vol. 12, no. 1, pp. 23-42.

3. Williamson O.E. Transaction-cost economics: the governance of contractual relations. Journal of Law and Economics, 1979, vol. 22, no. 2, pp. 233-261.

4. Jensen M.C., Meckling W.H. Theory of the firm: managerial behavior, agency costs and ownership structure. Journal of Financial Economics, 1976, vol. 3, no. 4, pp. 305-360.

5. Antonenko E.V. K raskrytiiu sushchnosti i klassifikatsii transaktsionnykh izderzhek [To disclosure of essence and classification of transaction expenses]. Bulletin of the South Ural State University. Series "Economics and Management", 2015, vol. 9, no. 1, pp. 74-80.

6. Eggertsson T. The role of transaction costs and property rights in economic analysis. European Economic Review, 1990, vol. 34, no. 2/3, pp. 450-457. 
7. Prietula M.J., Weingart L.R. Negotiation offers and the search for agreement. Negotiation and Conflict Management Research, 2011, vol. 4, no 2, pp. 77-109.

8. Robu V., Somefun D.J.A., Poutre J.L. Modeling complex multi-issue negotiations using utility graph. Proceedings of the Forth International Joint Conference on Autonomous Agents and Multi-Agent Systems, 2005, pp. 280-287.

9. Fujita K., Ito T., Klein M. An approach to scalable multi-issue negotiation: decomposing the contract space. Computation intelligence, 2014, vol. 30, pp. 30-47.

10. Abedin F., Chao K.-M., Godwin N. An agenda based multi issue negotiation approach. Journal of Ambient Intelligence and Humanized Computing, 2014, vol. 5, no. 4, pp. 455-473.

11. Fatima S.S, Wooldridge M., Jennings N.R. Multi-issue negotiation with deadlines. Journal of Artificial Intelligence Research, 2006, vol. 27, pp. 381-417.

12. Azgal'dov G.G., Kostin A.V., Sadovoi V.V. Kvalimetriia dlia vsekh [Qualimetry for everyone]. Moscow, Informznanie, 2012, $165 \mathrm{p}$.

13. Poliakova M.A. Razvitie teorii otsenki soglasovannosti tekhnicheskikh trebovanii na metalloproduktsiiu pri razrabotke normativnoi i tekhnicheskoi dokumentatsii [Development of the theory of assessment the coherence of technical requirements for metal products with the elaboration of regulatory and technical documentation]. Doctor's degree dissertation. Magnitogorsk, 2017, $235 \mathrm{p}$.

14. Poliakova M.A. Osobennosti primeneniia matematicheskikh modelei dlia otsenki stepeni dostizheniia konsensusa pri razrabotke trebovanii standartov [Peculiarities of mathematical models application for the estimation of consensus achievement degree during development the demands of standards]. International Research Journal, 2016, no. 11(53), part 4, pp. 104-109.

15. Rubin G.Sh., Kasatkina E.G. S-obraznaia matematicheskaia model' edinichnoi otsenki kachestva [S-shaped mathematical model of a single quality assessment]. Kachestvo v obrabotke materialov, 2014, no. 2, pp. 74-81.

16. Rubin G.Sh., Poliakova M.A., Gun G.S. Modelirovanie tekhnologicheskogo transformirovaniia na osnove S-obraznykh krivykh razvitiia [Modeling of the technological transformation based on S-shape curves of development]. Vestnik of Nosov Magnitogorsk State Technical University, 2015, no. 1, pp. $70-75$.

17. Azgal'dov G.G. Teoriia i praktika otsenki kachestva tovarov. Osnovy kvalimetrii [Theory and practice of product quality assessment. Fundamentals of qualimetry]. Moscow, Ekonomika, 1982, 256 p.

18. Danilova Iu.V. Sovershenstvovanie protsedury razrabotki standartov na metalloizdeliia formalizatsiei soglasovaniia pozitsii zainteresovannykh storon [Improving the procedure for the development of metal products standards by formalizing the coordination of stakeholders positions]. Ph.D. thesis. Magnitogorsk, 2016, $155 \mathrm{p}$.

19. MMK-Metiz, available at: http://mmk-metiz.ru (accessed 2 December 2014). 
20. Annual reports of MMK-Metiz, available at: http://www.mmkmetiz.ru/investor/reports/ (accessed 7 December 2015).

21. Alto Consulting Group, available at: http://alto-group.ru/otchot/ marketing/363-rynok-metizov-krepezhnyx-izdelij-tekushhaya-situaciya-i-prognoz-20142018-gg.html (accessed 5 December 2015)

Оригинальность $75 \%$

Получено 05.02.2018 Принято 01.03.2018 Опубликовано 29.06.2018

E.V. Antonenko, M.A. Polyakova, S.A. Fedoseev

\title{
ESTIMATION OF DEGREE OF COHERENCE BETWEEN CONSUMER AND MANUFACTURER REQUIREMENTS AS THE METHOD FOR TRANSACTION COST REDUCTION
}

\begin{abstract}
The definition of transaction costs arising due to enterprises' market interaction and including real and alternative losses incurred as a result of the interaction is offered. Transaction costs which emerge during negotiations between the consumer and the manufacturer of steel products are considered. It is noted that time spent by consumer and manufacturer of steel products for requirements' coherence is quite significant in practice. Therefore, the task of choosing approaches to reduce duration of such negotiations and transaction costs connected with these negotiations is an urgent one. One of such approaches based on the quantitative criterion is considered. The criterion evaluates the degree of consumer and manufacturer requirements' coherence. Mathematical models based on s-curves and the apparatus of qualimetry are proposed as one of the methods for determining this criterion. It is shown that it is necessary to take into account the features of product quality indicators standardization in regulatory and technical documentation for the development of such mathematical models. Principles for degree of coherence between consumer and manufacturer quantitative assessment are provided, namely: the assessment should be calculated as a function of a certain product quality indicator; the assessment should continually depend on the value of the product quality indicator; the assessment should have peculiar areas of intensive and slow growth. An illustration of how the proposed methods can significantly reduce the time of negotiations and consumer losses related with price increase is given.

Keywords: transaction costs, reducing losses, mathematical model, product quality, degree of requirements coherence.
\end{abstract}

Elizaveta V. Antonenko - Lecturer, Dept. of Customs, South Ural State University (national research university), Chelyabinsk, e-mail: Elizaveta.antonenko@susu.ru.

Marina A. Polyakova - Candidate of Technical Sciences, Associate Professor, Dept. of Processing Technology of Materials, Nosov Magnitogorsk State Technical University, e-mail: m.polyakova-64@mail.ru.

Sergey A. Fedoseev - Doctor of Technical Sciences, Professor, Dept. of Computational Mathematics and Mechanics, Perm National Research Polytechnic University, e-mail: fsa@gelicon.biz.

Received 05.02.2018 Accepted 01.03.2018 Published 29.06.2018 